\title{
4G LTE and LTE-Advanced using Simulation
}

\author{
Kirti kapse And Prof. Raju kamble \\ Indira college of engineering and management parandhwadi pune.
}

\begin{abstract}
In this paper, the performance of Long term evolution (LTE) based on orthogonal frequency-division multiple access (OFDMA) is investigated which uses multiple input, multiple output technique (MIMO). This paper gives an peak to power average ratio (PAPR) analysis of MIMO performance along with OFDMA modulation using Matlab of the next generation telecommunication network LTE $3^{\text {rd }}$ Generation partnership project (3GPP). The downlink is based on orthogonal frequency-division multiple access at the physical layer, while the uplink is based on single carrier frequency-division multiple access. The PAPR reduction to achieve a low clipping rate which is actually done in SC-FDMA, as clipping is low the efficiency is also low which provide great benefit in power consumption. Thus LTE can transmit a packet efficiently with low latency and as well as without transmission error. This paper gives the comparison of LTE release 8 and LTE release 10.It provides communication with a terminal, moving up to $500 \mathrm{~km} / \mathrm{h}$.
\end{abstract}

Index Terms: $3 G P P, L T E, M I M O, P A P R, S C-F D M A$.

\section{INTRODUCTION}

In times when mobile devices are getting more popular the mobile network are becoming more and more advanced too. Websites are not the same they used to be 10 years ago. Now in recent they are consisting of with high quality pictures, animation, flash application and many more. With this the demand for mobile internet grew significantly [1]. According to opera's web browser the number of pages viewed has raised from about 23 billion in January 2010 to 177 billion pages in November 2013[2]. This means leading to an increase of amount of data. Nowadays there are not only simple telephonic calls and short messages anymore. There is a great demand of video telephony, multimedia messaging and email facilities and much android Application for online accessing. Over the past years the promise of mobile broadband has become reality as many tens of millions of users have actively started using Smartphone, tablets, net books, and laptops with wireless connection [3]. These examples shows the huge demand for higher transfer rate, better availability, higher speed for mobile internet Connections. The communication industry has been formulating new stands to efficiently deliver high Speed broadband mobile access in a single air interface and network architecture at low cost to operate and end users. Two standards IEEE 802.16 they are worldwide interoperability for microwave access and 3GPP long term evolution are leading the pack towards forming the next generation of mobile network standards.

\section{A. Overview}

Currently the mobile network infrastructure comprises overlay networks including 2G and 3G technologies [4]. Global system for mobile communication (GSM) $2^{\text {nd }}$ generation mobile network and provides circuit switched communication. IT was enhanced by general packet Radio services also known as $2.5 \mathrm{G}$ and enhanced data rate for GSM evolution (EDGE) also known as $2.75 \mathrm{G}$ providing IP functionality and data transfer rate up to $13 \mathrm{mbps}$ in the downlink and $653 \mathrm{kbps}$ on the uplink [5]. These are all family of $2 \mathrm{G}$ to $2.75 \mathrm{G}$ wireless system. Similarly we have family of $3 \mathrm{G}$ wireless standard there is WCDMA/UMTS has data rate of $384 \mathrm{kbps}$ also CDMA has the same data rate for voice call and packet data. Again we have 3.5G HSDPA/HSUPA has data rate of 5-30 mbps in this category we have 1XEVDO. LTE Release 8 is one of the primary broadband technologies based on OFDMA, which is currently being commercialized. LTE Release 8, provides improved system capacity and coverage, high peak data, low latency, reduced operating costs, multi-antenna support, flexible bandwidth operation and seamless integration with existing systems. LTE Release 8 supports much higher peak rates, higher throughout and coverage and lower latencies, resulting in a better user experience [6].

\section{LTE}

Long term evolution (LTE) in 3GPP is the latest technology in cellular network that is being standardized. It is considered as a $3.9 \mathrm{G}$ cellular system. The name LTE comes from the evolved universal terrestrial radio access network [E-UTRAN]. It is based on all internet protocol frame work that is not limited by past design. The peak data rate is also increased, with the downlink having $100 \mathrm{Mbps}$ and the uplink having $50 \mathrm{Mbps}$. LTE uses 
Multiple-input, Multiple-output (MIMO) technique. The downlink is based on orthogonal frequency division multiple access (OFDMA) at the physical layer, while the uplink is based on single carrier frequency division multiple access (SC-FDMA). Each radio frame is $10 \mathrm{~ms}$ long and can be divided into sub frame each of $1 \mathrm{~ms}$ duration. Each sub frame can be further divided into two slots, each of $0.5 \mathrm{~ms}$ duration. LTE also uses transmission power control (TPC) and Adaptive modulation and coding (AMC). Access to the network is dependent on the physical layer, medium access control, radio link control, packet data convergence protocol, and radio resource control. Tight interaction among these building blocks is necessary to transmit a packet efficiently with low latency and as well as without transmissions error [7].

\section{A.Overview}

LTE is a long term evolution support data rate of $100 \mathrm{mbps}$ in downlink and $50 \mathrm{mbps}$ in uplink both reachedwith $20 \mathrm{MHz}$ spectrum. LTE is available for both FDD and TDD modes. LTE is $3 \times$ faster than HSPA+, even though both are often branded $4 \mathrm{G}$ with faster pings. LTE offers vastly improved performance for VOIP services like Skype. Ping is significant because a high ping reduces the impact of fast download speeds, as every packet of information download needs to be acknowledged.

\section{B. Features}

LTE uses orthogonal frequency division multiple access (SCFDMA) in the uplink [8]. It has evolved EDGE capabilities available to significantly increases EDGE throughput rates and announced deployment. HSPA+ peak speeds further increased to peak rates of $42 \mathrm{mbps}$ based on Release 8. LTE introduced for next generation throughput performance using 2x2 MIMO. Advanced core architecture available through EPC primary for LTE, but also for HSPA+, providing benefits such as integration of multiple network types and flatter architecture for better latency performance. LTE enhancement such as 4x MIMO and dual carrier available. Now in 2012 later LTE-Advanced potentially deployed in initial stages and HSPA+ with MIMO and quad carrier available, new services implemented in the packet domain.

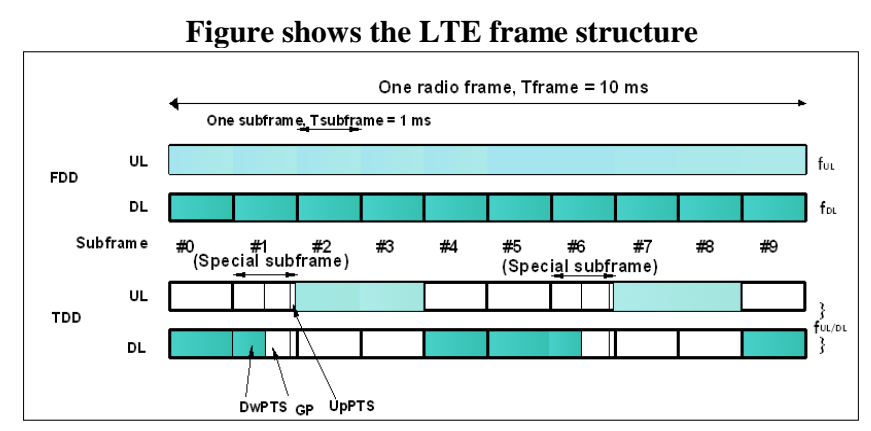

Fig 1. Shows LTE frame structure

Fig 1 shows the LTE frame structure. It supports both FDD and TDD also difference between two is the presence of a sub frame to provide guard time for downlink to uplink switching. Because of using OFDM, LTE can use channel dependent scheduling in both times as well as in frequency domain. For each $1 \mathrm{~ms}$ sub frame in which user are allowed to transmit. In the frequency domain, the scheduler granularity is $180 \mathrm{KHz}$. Both downlinks and uplink transmission are controlled by the scheduler located in the base station. The scheduler is the key element and determines the overall downlink system performance. The instantaneous channel quality at the terminals is find out and again fed back to the base station as per sub frame. In the uplink the terminal can be configured to transmit a sounding reference signal which may be used for uplink channel dependent scheduling.

\section{Advanced features of LTE:}

\section{a) Time-Frequency Domain Scheduling:}

In LTE, time and frequency domain channel-dependent scheduling is supported for both uplink (PUSCH), and downlink (PDSCH). This is achived through acquiring the CQIs of the respective channel. By doing so, multiuser diversity gain is achived.

\section{b) Link Adaptation:}

In LTE, downlink supports QPSK and 16-QAM, and 64-QAM modulation. In uplink, QPSK and 16-QAM are supported, with 64-QAM being optional. LTE supports various link adaptation including transmission power control, adaptive modulation, and coding as well as adaptive bandwidth allocation. 
c) MBMS:

LTE supports MBMS through single frequency network(SFN). All cell provide the broadcasting using the same set of resources. The signal coming from the surrounding cells are treated as multipled delayed version using long CP.

\section{d) MIMO:}

To achieve a 100 Mbps peak data rate in downlink transmission over a 20-MHz bandwidth, two independent data streams are multiplexed through $2 \times 2$ MIMO or $4 \times 2$ MIMO system.

\section{LTE -ADVANCED}

LTE Release 10, also known as LTE-Advanced includes all the features of Release 8/9 and adds new features which enhanced multi antenna support. In order to achieve $1 \mathrm{~Gb} / \mathrm{s}$ peak data rate in future IMT-Advanced mobile systems, carrier aggregation technology is introduced by the $3 \mathrm{GPP}$ to support very high data rate transmission over wide frequency bandwidth in its LTE-Advanced standards [9]. One of the most important LTE-Advance benefits is the ability to take advantage of advanced topology networks, optimize heterogeneous network with a mix of macro cell with low power nodes such as picocells, femtocells and new relay nodes. The next significant performance leap in wireless network will come from making the most of topology, and brings the networks closer to the user by adding many of this low power nodes-LTE Advance further improves the capacity and coverage, and ensures user fairness. LTE- Advance also introduces multicarrier able to use ultra wide bandwidth, up to $100 \mathrm{MHz}$ of high data rates.

\section{A.Overview:}

LTE officially known as 3GPP Release 10 and beyond Release 12 is still in development by the 3GPP LTE-Advanced, which can be used as a real 4G-telecommunication network. LTE-Advance support higher transfer rates up to 1 gbitps in downlink and 500 mbps for uplink for low mobility.

Downlink: Extended SU-MIMO (up to 8 antenna).

Support for carrier aggregation with 5

carrier component.

Release 9 PRS.

Release 10 UE specific.

Release 10 CSI RS

Uplink: Uplink SU-MIMO for PUSCH.

Uplink spatial diversity.

Support for carrier aggregation with 5 carrier

component.

Cluster SC-FDMA.

Possibility simultaneous transmission for

PUCCH and PUSCH.

IV. COMPARISON OF LTE AND LTE-ADVANCED

\begin{tabular}{|l|l|l|}
\hline Features & LTE & LTE-Advanced \\
\hline Generation & $3.9 \mathrm{G}$ & $4 \mathrm{G}$ \\
\hline First release & 2009 & 2011 \\
\hline Physical layer & DL= OFDMA & DL= OFDMA \\
& UL= SCFDMA & UL= SCFDMA \\
\hline Duplex mode & Time and Frequency division & Same as LTE \\
& duplex & \\
\hline User mobility & Up to $350 \mathrm{kmph}$ & Same as LTE \\
\hline Coverage & Up to $100 \mathrm{~km}$ & Same as LTE \\
\hline Transmission bandwidth & $\mathrm{DL}=20 \mathrm{MHz}$ & $\mathrm{DL}=100 \mathrm{MHz}$ \\
& $\mathrm{UL}=20 \mathrm{MHz}$ & $\mathrm{UL}=40 \mathrm{MHz}$ \\
\hline Peak data rate & $\mathrm{DL}=300 \mathrm{Mbps}$ & $\mathrm{DL}=1 \mathrm{Gbps}$ \\
& $\mathrm{UL}=75 \mathrm{Mbps}$ & $\mathrm{UL}=500 \mathrm{Mbps}$ \\
\hline VoIP capacity other qualities & 200 active user percell in 5 & 3 times higher as compared to \\
& $\mathrm{MHz}$ & LTE \\
\hline
\end{tabular}

Table 1: Comparison of LTE and LTE-Advanced. 


\section{BLOCK DIAGRAM OF SC-FDMA AND OFDMA WITH RECEIVER AND TRANSMITTER:}

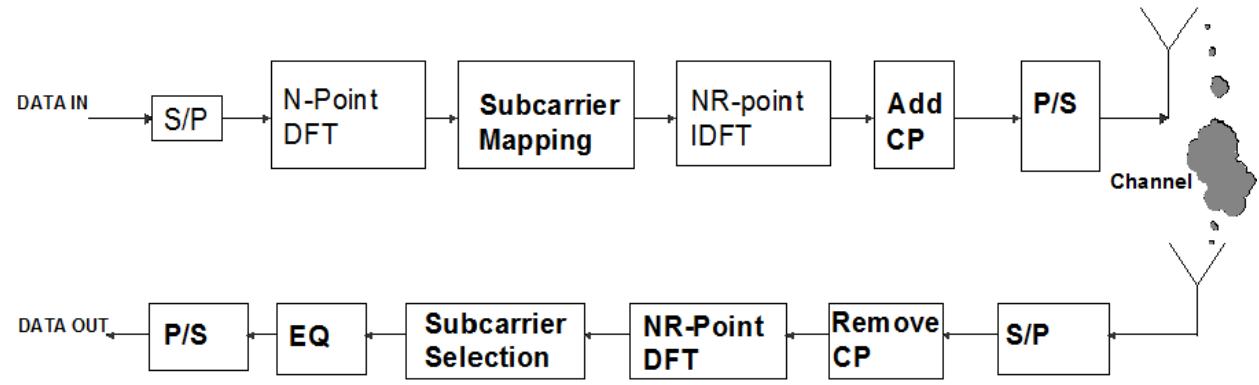

Figure 3: Block diagram of SC-FDMA with transmitter and receiver.

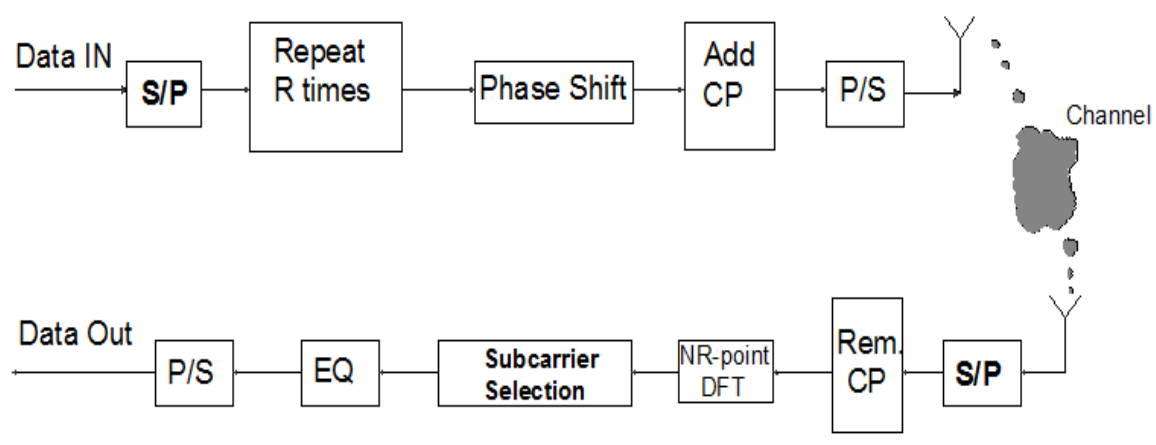

Figure 3: Block diagram of OFDMA with transmitter and receiver

VI. Matlab Results:
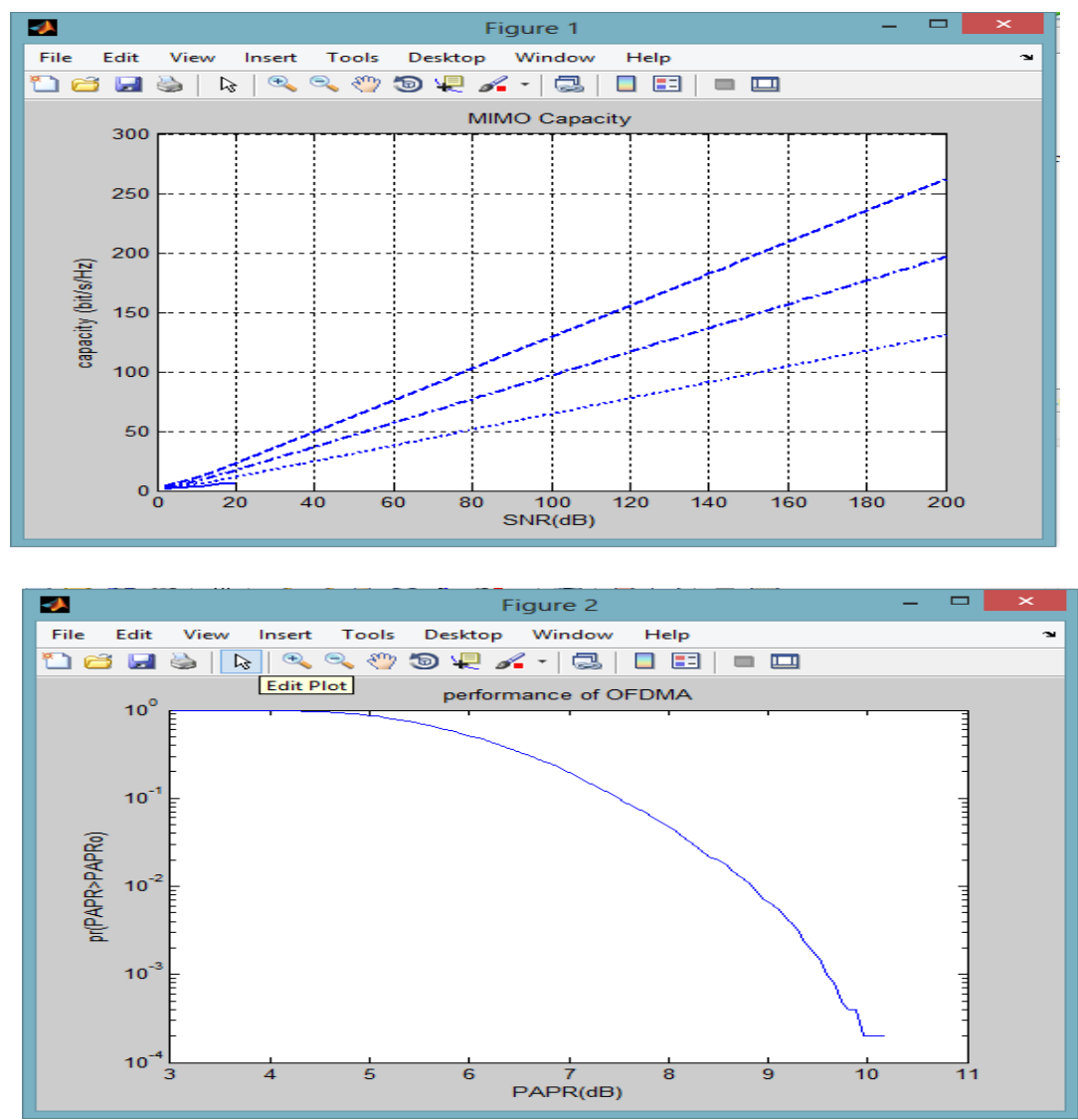


\section{CONClusion OF THE PAPER:}

LTE successfully run all over the world wide and its growth continue unabated. With this LTE is expected to support different types of application such as: Web browsing, File transfer protocol (FTP), Video streaming, Music streaming, Voice over IP, network gaming, Real time video, Push to talk (PTT) and Push to view (PTV). Also with this SC-FDMA is the better option for cellular uplink in LTE, because of its higher efficiency due to low PAPR, its lower sensitive to frequency offset consumption in the uplink, and OFDMA in the downlink which have been shown in matlab figure. We can minimize the PAPR ratio with respect to SC-FDMA and OFDMA. In this paper we have plotted the CCDF that is known as Complementary cumulative distribution function of the amplitude of a signal sample is given by,

$\mathrm{F}(\mathrm{Z})=1-\mathrm{e}^{\mathrm{Z}}$

The CCDF of the PAPR of a data block with Nyquist rate sampling is,

$\mathrm{P}(\mathrm{PAPR}>\mathrm{Z})=1-\mathrm{P}(\mathrm{PAPR} \leq \mathrm{Z})$

$=1-\mathrm{F}(\mathrm{Z})^{\mathrm{Z}}$

$=1-\left(1-\mathrm{F}(\mathrm{Z})^{\mathrm{N}}\right)$

Assuming that $\mathrm{N}$ times domain signal samples which are mutually independent and exclusive.

\section{References:}

[1]. Emad kazi, Rajan pillai, uzair Qureshi, Awab faikh, Long term evolution. IOSR journal of Electronics and communication engineering sept- Oct 2013.

[2] .State of the mobile web, October 2013, "Opera tech report, oct-2013.

[3]. Rysavy research, Transition to 4G, 3GPP broadband evolution to IMT-Advance sept-2010.

[4]. Dieter eberle, LTE Vs WIMAX, 4G telecommunication network Berlin institute of technology Germany sept-2010.

[5]. MOHD Azharuddin, Comparison between LTE Advance Vs WImax release 2.0 for communication network

[6]. G Yuan, X Zhang, W Wang, Y Yang, Carrier Aggregation-LTE Advanced Mobile communication system, IEEE communication Magazine 2010.

[7]. Wireless Broadband network, D. Chong Wong, p yong Kong, Y chang Liang, K chaing chua, and J w mark 2009

[8]. MOHD Azharuddin, Comparison between LTE Advance Vs WImax release 2.0 for communication network.

[9]. R. Nossenson, "Long term evolution network architecture in microwave communication engineering magazine, IEEE international conference in 2009 . 